\title{
Decoding real space bonding descriptors in valence bond language
}

\author{
Ángel Martín Pendás* and Evelio Francisco \\ Departamento de Química Física y Analítica. Universidad de Oviedo. Spain. \\ E-mail: ampendas@uniovi.es
}

\begin{abstract}
Real space bonding descriptors are orbital invariant indices that can be obtained independently of the theoretical framework used to compute a given wavefunction. Here we show how to use them to read in real space some widely used concepts in Valence Bond (VB) theory, such as ionic/covalent characters or covalent-ionic resonance energies. All of these are essential ingredients used when building VB chemical insight. Electron number distribution functions are employed to directly map ionic and covalent weights with real space delocalization indices. We show that all covalency emerges in position space from the fluctuation of electron populations or, in other words, from covalent-ionic resonance. The reasons why this is not so in non-orthogonal VB are examined. A simple ionic character index that maintains the essence of its VB equivalent is defined and examined in simple model systems. The conclusions of this work ease travelling among the sometimes conflicting molecular orbital, real space, and valence bond interpretations in chemical bonding theory.
\end{abstract}

Historical as well as computational reasons have been put forward over the years to explain the predominance of molecular orbital (MO) ${ }^{1}$ theory over the valence bond (VB) approach. ${ }^{2-4}$ Given that chemical interpretations of quantum mechanical results heavily depend on theoretical

${ }^{*}$ To whom correspondence should be addressed 
frameworks, it is not unexpected that insights on chemical bonding issues may get very different answers when the MO or VB interpretation toolkits are used. The pervasiveness of MOs does also explain why MO arguments dominate chemical bonding reasoning. Be that as it may, theoretical and computational advances in the last decade are changing the scenario, and as VB calculations become more and more feasible, so do VBish interpretations permeate the literature. An example of new concepts that emanate from the new VB methods is the charge shift bond (CSB), ${ }^{5}$ which has been publicized as a genuine third chemical bond category besides the traditional covalent and ionic classes. Central to the CSB concept is the covalent-ionic resonance energy, which has no direct MO counterpart although several tricks can be used to reframe it in the traditional MO context. 1

Another conceptual edifice in chemical bonding theory exists that provides descriptors which are invariant under orbital transformations. These indices are thus independent on whether MO or VB are used to construct a given wavefunction. We are referring to the real (or position) space approaches to the chemical bond, which are collectively gathered under the quantum chemical topology (QCT) umbrella. In QCT, reduced density matrices (RDMs) are the pillars used to build chemical insight. Through them, space may be partitioned into regions bearing chemical information, like when the quantum theory of atoms in molecules ${ }^{6}$ (QTAIM) is employed to provide atomic domains. Once a space partitioning is at hand, an energetic decomposition is also available through the interacting quantum atoms approach ${ }^{7,8}$ (IQA), and information about how electrons distribute themselves in those real space fragments can be recovered through electron number distribution functions ${ }^{9-11}$ (EDFs).

A large body of knowledge is now available that relates QCT with the standard MO paradigm. ${ }^{12}$ QCT provides both local and global bonding indicators. The former include properties at distinguished points in space, usually the critical points of a scalar field with chemical meaning, like the electron density. Global indicators are obtained by integrating operator densities over the spatial domains, and they include energy components, atomic net charges or delocalization indices, which are the real space analogues of bond orders. Although some works have been published, ${ }^{13}$ not 
much is known about the relation between QCT and VB. Here we provide some decoding tips regarding the concepts of covalent and ionic weights, as well as on the meaning of covalent-ionic resonance in real space.

To do so we will consider two-center two-electron model systems together with IQA and EDF concepts which we briefly review. Given a QTAIM partition of space into quantum atoms $A_{i}, R^{3}=$ $\bigcup_{i} A_{i}$, IQA writes the binding energy of a system as sum of intra- and interatomic contributions, known as deformation and interaction energies, respectively: 7,8

$$
E_{\text {bind }}=\sum_{A} E_{d e f}^{A}+\sum_{A>B} E_{i n t}^{A B}
$$

As atomic deformation energies are generally destabilizing, ${ }^{7,8}$ chemical bonding is driven by how two given atoms or fragments interact with each other. The interatomic interaction energy, $E_{\text {int }}^{A B}$ can be further decomposed into an ionic or classical term, $E_{c l}^{A B}$, and a covalent or exchange-correlation one, $E_{x c}^{A B}: E_{\text {int }}^{A B}=E_{c l}^{A B}+E_{x c}^{A B}$. We stress at this point that the ionic component $E_{c l}^{A B}$ is the total classical electrostatic interaction energy of all the particles contained in region $A$ with those lying in region $B$. Except in the case of intermolecular interactions where dipole, quadrupole or further multipolar terms may provide (weak) stabilizations, $E_{c l}$ can only lead to strong bonding when there is substantial net charge transfer between the two regions. It is in this charge-transfer sense that the term ionic is used in IQA. Since we will be using here this word also to consider the ionic structures of VB theory, which may or may not be accompanied by net charge transfer, we will take care not to confuse both meanings. $E_{x c}^{A B}$ is obtained by computing the interatomic Coulombic energy of pairs described by the the exchange-correlation density $\rho_{x c}\left(\boldsymbol{r}_{1}, \boldsymbol{r}_{2}\right)=\rho\left(\boldsymbol{r}_{1}\right) \rho\left(\boldsymbol{r}_{2}\right)-\rho_{2}\left(\boldsymbol{r}_{1}, \boldsymbol{r}_{2}\right)$, which defines how the pair density $\rho_{2}$ deviates from the independent product of one-electron densities $\rho(\boldsymbol{r}):$

$$
E_{x c}^{A B}=-\int_{A} d \boldsymbol{r}_{1} \int_{B} d \boldsymbol{r}_{2} \frac{\rho_{x c}\left(\boldsymbol{r}_{1}, \boldsymbol{r}_{2}\right)}{r_{12}}
$$

If in the above expression we exclude the $r_{12}^{-1}$ term we get the delocalization index $\mathrm{DI}(A, B)=$ $\delta^{A B}=2 \int_{A} d \boldsymbol{r}_{1} \int_{B} d \boldsymbol{r}_{2} \rho_{x c}\left(\boldsymbol{r}_{1}, \boldsymbol{r}_{2}\right)$. The DI is a measure of the extent of inter-domain electron de- 
localization, and may be shown to transform smoothly into the Wiberg-Mayer bond order ${ }^{14}$ when a Mulliken instead of a QTAIM condensation is performed. It can also be shown to be simply the covariance of the electron number probability distribution, $\delta^{A B}=-2 \operatorname{cov}\left(n_{A}, n_{B}\right)$, where $n_{A}, n_{B}$ count the number of electrons in the $A, B$ regions, respectively. This last relation provides a first link between chemical bonding ideas and the statistics of the electron distribution.

Let us now restrict ourselves to a two-center (domains $A$ and $B$ ) closed-shell two-electron bond for the sake of simplicity. We will specifically consider the $\mathrm{H}_{2}$ and $\mathrm{LiH}$ molecules, where in the last case the $\mathrm{Li}$ core electrons are treated as chemically inert. It is clear ${ }^{15}$ that there are only three possible spinless distributions of the electrons in the two domains: two electrons in the left $(A)$ domain and none in the right one, one in $A$ and one in $B$, or the two in the right $(B)$ domain. We call them real space resonance structures (RSRSs). Their probabilities will be $p(2,0), p(1,1)$, and $p(0,2)$, respectively, fulfilling an obvious sum rule $p(2,0)+p(1,1)+p(0,2)=1$. General expressions for the probability of a given partition of an $N$ electron system divided in $m$ regions can be found. ${ }^{9,15}$ If spin is taken into account, the $(1,1)$ distribution gives rise to two spin-resolved structures, with probabilities $p(\uparrow, \downarrow)$ and $p(\downarrow, \uparrow)$ and the total number of structures is now four. Any possible spinless electron distribution can be mapped onto a point in a triangular diagram in which each corner corresponds to a pure resonance structure with $p=1 .^{15}$

Spatial resonance structures correspond closely to their VB counterparts. For a two-electron homodiatomic like $\mathrm{H}_{2}$, the simplest Heitler-London covalent structure is written as

$$
\psi_{\text {cov }}=\frac{1}{\sqrt{2\left(1+S^{2}\right.}}\left(\phi_{A}(1) \phi_{B}(2)+\phi_{B}(1) \phi_{A}(2)\right) \frac{1}{\sqrt{2}}(\alpha(1) \beta(2)-\beta(1) \alpha(2))
$$

where we have simplified the description of electron coordinates, $\phi$ is a $1 s$ hydrogenic function, and $S=\left\langle\phi_{A} \mid \phi_{B}\right\rangle$ is the standard atomic orbital overlap. Similar expressions can be written down for the two (equivalent) ionic structures,

$$
\psi_{\text {ion }}^{C}=\phi_{C}(1) \phi_{C}(2) \frac{1}{\sqrt{2}}(\alpha(1) \beta(2)-\beta(1) \alpha(2))
$$


with $C$ being either $A$ or $B$. A typical VB wavefunction is constructed by a linear combination of the above, $\psi=\lambda_{c o v} \psi_{c o v}+\mu_{i o n}^{A} \psi_{i o n}^{A}+\mu_{i o n}^{B} \psi_{i o n}^{B}$. Standard notation would write $\psi_{c o v}$ as $\mathrm{H}_{A} \cdot \cdot \mathrm{H}_{B}$, and $\psi_{i o n}^{A}$ as $\mathrm{H}_{A}^{-} \mathrm{H}_{B}^{+}$. In $\mathrm{H}_{2}, \psi_{\text {cov }}$ provides a reasonable description of $\mathrm{H}_{2}$ binding, with $D_{e} \approx 96$ $\mathrm{kcal} / \mathrm{mol} .{ }^{5}$ Mixing the ionic structures in $\psi$ leads to a covalent weight $w_{c o v}$ of about 0.76 and a much better $D_{e}=105 \mathrm{kcal} / \mathrm{mol}$, the difference in both energies being nothing but the covalentionic resonance energy. In $\mathrm{F}_{2}$, a prototype of $\mathrm{CSB}$, both $\psi_{c o v}$ and $\psi_{\text {ion }}$ provide repulsive energy curves, and only when they are allowed to mix a stable molecule is recovered. ${ }^{5}$ It is customary to imagine, and quote, covalent structures as those sharing one electron in each atom, in agreement with the Lewis pair concept, while ionic terms are thought as those in which one of the electrons of the pair has been transferred to the other atom.

The equal number of VB and real space resonance structures is obviously not casual. There is a clear one-to-one correspondence among them, and the VB covalent structure is to be associated with the $(1,1)$ distribution as well as the VB ionic structures match the $(2,0)$ and $(0,2)$ ones. This is the starting point of several important conclusions we now detail.

A first relevant insight comes from the relation between the DI or $E_{x c}$, which is the only stabilizing interaction term in a homodiatomic molecule, and the fluctuation of the electron populations. There is no bonding without fluctuation. At dissociation, for instance, the probability distribution ${ }^{15}$ in $\mathrm{H}_{2}$ has obviously only one component, and $p(1,1)=1$. Since only one of the three RSRSs is populated there is no fluctuation at all in the electron populations, and the DI and $E_{x c}$ vanish. The same can be said if a molecule displays, for instance, $p(2,0)=1$ and one electron has been completely transferred from the right to the left basin. This system may be stabilized by electrostatics, but both $E_{x c}$ and the covalent DI vanish. There is no covalency without population fluctuations. Were the $p(1,1)$ probability equal to one in $\mathrm{H}_{2}$ at its equilibrium distance and its covalent energy would also vanish. For covalency to occur in this molecule we need the ionic structures populated. This means that the role of the covalent structures in VB has to be reinspected carefully.

How can the covalent VB structure in $\mathrm{H}_{2}$, described by $\psi_{c o v}$, lead to such a good description of the molecule in the light of the above insights? The answer is disappointingly simple. Fig. Figure 1 


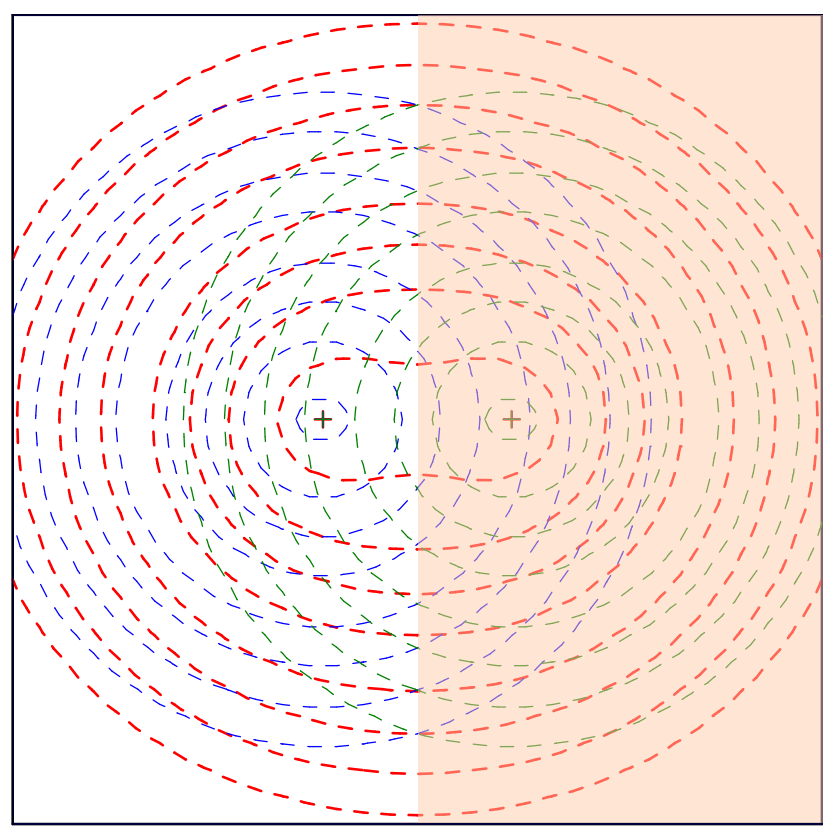

Figure 1: Isolines of the total electron density (red) in $\mathrm{H}_{2}$ at the CAS[2,2]//6-311++G* theoretical geometry on one of the planes containing the nuclei. Isocurves of the left $\phi_{A}^{2}$ and right $\phi_{B}^{2}$ densities are also shown in blue and green, respectively. The two real space atomic domains are separated by the perpendicular midplane intersecting the internuclear axis, and the position of the nuclei is pointed out with crosses.

shows how deeply the two $\mathrm{H}$ atomic densities interpenetrate each other $(S \approx 0.68)$. This means that, in real space, even $\psi_{c o v}$ shows very large $p(2,0)$ and $p(0,2)$ probabilities, i.e. the VB covalent structure includes effectively ionic contributions, exactly those that cause a non-vanishing fluctuation of the electron population. How large this effect is depends of $S$, the atomic overlap. This returns us to the no-bonding without overlap motto that summarizes the know-how of chemical bonding in Fock space. Our analysis permits to disentangle the actual role of overlap. A similar conclusion about the role of $p(2,0)$ and $p(0,2)$ may be reached by using orthogonal flavors of VB. ${ }^{2}$ In these VB implementations, any covalent structure effectively includes all the ionic ones.

Table 1: Probabilities and DIs of the spin-resolved resonance structures associated to the ideal VB and HF models of a homonuclear two-center two-electron bond

\begin{tabular}{lcccc|cccc}
\hline State & $p(\uparrow, \downarrow)$ & $p(\downarrow, \uparrow)$ & $p(\uparrow \downarrow, 0)$ & $p(0, \downarrow \uparrow)$ & $\mathrm{DI}^{\sigma \sigma}$ & $\mathrm{DI}^{\sigma \sigma^{\prime}}$ & $\mathrm{DI}$ & $p(0,2)+p(2,0)$ \\
\hline VB Covalent & $1 / 2$ & $1 / 2$ & 0 & 0 & 1 & -1 & 0 & 0.0 \\
HF & $1 / 4$ & $1 / 4$ & $1 / 4$ & $1 / 4$ & 1 & 0 & 1 & 0.5 \\
VB Ionic & 0 & 0 & $1 / 2$ & $1 / 2$ & 1 & 1 & 2 & 1.0 \\
\hline
\end{tabular}


A second conclusion we want to point out is that in real space all covalent bonding comes from the covalent-ionic resonance. Since any system in which only one resonant structure is populated (with $p=1$ ) has no covalent energy component, it is actually the resonance among several of the structures that provides covalency. Usually the population of the $(1,1)$ term is non-zero, but it is in principle possible that pure ionic resonance, in which only the $(2,0)$ and $(0,2)$ structures fluctuate/resonate, may stabilize a system alone. All this has important consequences on the possibility to define the CSB in an orbital invariant manner, but we will defer this investigation which will be offered in a subsequent publication.

We now turn to propose a quantification of VBish concepts, like covalent and ionic weights, together with their role in determining bond orders or covalent energies from QCT descriptors. Table Table 1 contains the probabilities of the spin-resolved resonance structures associated to ideal models, in which electrons are associated to atomic domains with probabilities according to the chemical image of each of the models. The Hartree-Fock row is easy to write down. ${ }^{15}$ In a HF two-electron system opposite spin electrons are statistically independent so they delocalize freely giving rise to identical probabilities for the four possible spin-spatial distributions. It should be taken into account that any two-electron density or descriptor can be spin-resolved into same spin (Fermi) and opposite spin (Coulomb) contributions. For instance, the DI can be obtained as $\mathrm{DI}=\mathrm{DI}^{\sigma \sigma}+\mathrm{DI}^{\sigma, \sigma^{\prime}}$, where $\mathrm{DI}^{\sigma \sigma}=\mathrm{DI}^{\alpha \alpha}+\mathrm{DI}^{\beta \beta}$ and $\mathrm{DI}^{\sigma, \sigma^{\prime}}=\mathrm{DI}^{\alpha \beta}+\mathrm{DI}^{\beta \alpha}$. Similarly, we can calculate these DI components by computing spin-resolved covariances, as in $\mathrm{DI}^{\sigma \sigma}=-2\left(\operatorname{cov}\left(n_{A}^{\uparrow}, n_{B}^{\uparrow}\right),+\operatorname{cov}\left(n_{A}^{\downarrow}, n_{B}^{\downarrow}\right)\right)$ The models of Table Table 1 allow for a straightforward statistical calculation of all the DI components without any explicit quantum mechanical computation. These are also contained in the Table. As it can be seen, independently of the model, $\mathrm{DI}^{\sigma \sigma}=1$. This is actually so for any theoretical model of a $2 \mathrm{c}-2 \mathrm{e}$ homosystem that provides a probability $1 / 2$ of finding the alpha (or the beta) electron in each center. Since this is guaranteed by the singlet nature of the state under scrutiny, i.e. by spin entanglement, we can also consider $\mathrm{DI}^{\sigma \sigma}$ as measuring spin entanglement, or as effectively counting the number of spin coupled pairs. This is the reason why this index has been advocated as a better bond order descriptor than the DI 
itself. ${ }^{16}$ We show here that $\mathrm{DI}^{\sigma \sigma}$ does not reflecting bonding, but spin entanglement. Notice that each of the alpha or beta subcomponents of $\mathrm{DI}^{\sigma \sigma}$ determines how the electron spin delocalizes over the centers independently of the other. The Coulomb component describes the statistical correlation of opposite spin electrons. In the VB covalent model that corresponds to $\mathrm{H}_{2}$ at dissociation the alpha-beta pair is negatively correlated. If an alpha electron is in one center, its beta companion is in the other, and viceversa. Since there are no spin terms in the Coulomb Hamiltonian, the energy does only feel true spatial (not spin) delocalization, so that the spinless DI vanishes in the VB covalent state. In the HF approximation the effective electron correlation vanishes, and in the VB ionic model the correlation is positive. One alpha electron in one center implies that the beta one is also in that center. A direct way to check that $\mathrm{DI}^{\sigma \sigma}=1$ is to recognize that $\rho_{2}$ has only $\sigma \sigma^{\prime}$ components in a two electron system, so that the same spin component comes only from the integration of the Coulomb part of the exchange correlation density, $\rho_{x c}^{\sigma \sigma}(1,2)=\rho(1) \rho(2) / 2$. Integrating this expression leads to $\mathrm{DI}^{\sigma \sigma}=N_{A} N_{B}$, where $N_{A}$ is the average electron population of center $A$. In the homonuclear case $N_{A}=N_{B}=1$, independently of the internuclear distance or the level of theory.

A real space descriptor measuring the contribution of the VBish ionic terms in a given wavefunction is now easy to construct. A look to Table Table 1 shows that the $i=p(0,2)+p(2,0)$, the direct percentage of real space ionic contributions, behaves as desired, being zero for the VB covalent state, $50 \%$ in the HF state, and $100 \%$ in the purely ionic resonance. This index is clearly sensed by the DI. In the general heteroatomic case one can easily show that

$$
i=1-N_{A} N_{B}+\frac{\delta^{A B}}{2}=1-\frac{1}{2}\left(\delta^{A B, \sigma \sigma}-\delta^{A B, \sigma \sigma^{\prime}}\right) .
$$

Of course, a covalent contribution $c=1-i$ appears also from this definition. Notice that in the homoatomic case $i=\delta^{A B} / 2$. For instance, it is well known that the change of the delocalization index in $\mathrm{H}_{2}$ is sigmoidal with the $\mathrm{H}-\mathrm{H}$ distance, ${ }^{17}$ so this is also the way in which $i$ changes.

Fig. Figure 2 shows the evolution of $i$ with internuclear distance in $\mathrm{H}_{2}$. At equilibrium the ionic 


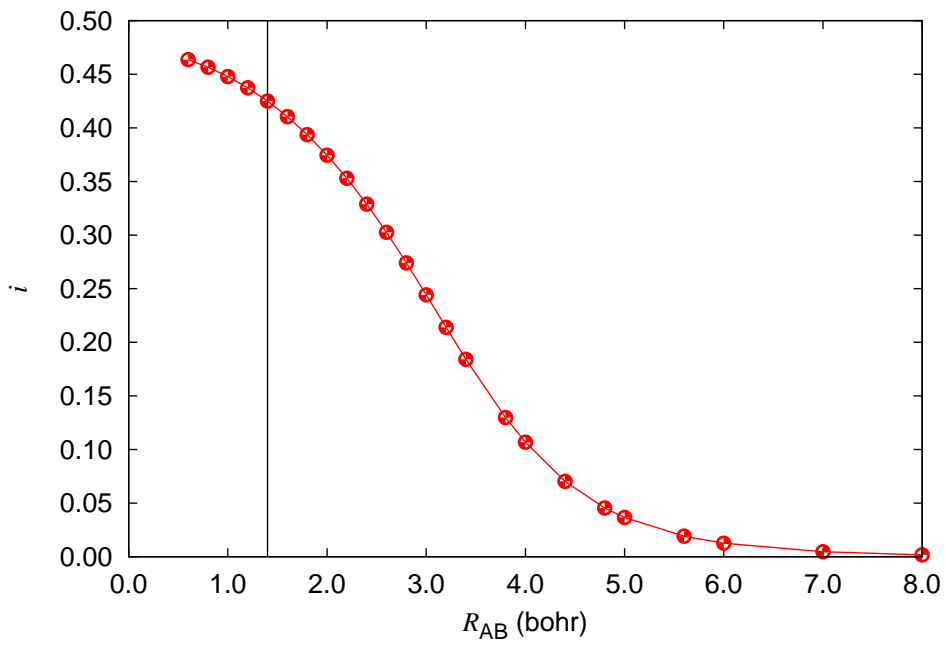

Figure 2: Ionic index $i$ for $\mathrm{H}_{2}$ computed at the FCI/cc-pVTZ level as the $\mathrm{H}-\mathrm{H}$ distance increases. The equilibrium distance is marked by a vertical line.

contribution for the full CI wavefunction is close, but smaller than the HF 50\% value. If we compare this with the aforementioned measure of the covalent weight in a typical VB calculation we can now understand why our ionic weight is considerably higher and much closer to the canonical HF percentage. As commented, even the covalent VB structure hides much ionic delocalization due to the large atomic orbital overlap. This is completely decoupled in the real space $i$. Since symmetry imposes the $p(2,0)$ and $p(0,2)$ weights to be equal, they can also be obtained from the Figure.

Fig. Figure 3 contains the same type of data for $\mathrm{LiH}$. As it can be seen, $i$ is very large at equilibrium, close to 0.9 . Contrarily to $\mathrm{H}_{2}, i$ is now completelly dominated by only one of the two $p(2,0)$ and $p(0,2)$ components, the one transferring electrons from the $\mathrm{Li}$ atom to the $\mathrm{H}$ moiety. As we noticed years ago, ${ }^{18}$ this is an electron charge transfer process, so that $i$ closely follows the curve of the net charge of the $\mathrm{Li}$ atom, $Q(\mathrm{Li})$. $\mathrm{LiH}$ is an interesting system from the VB perspective. A modern non-orthogonal VB calculation performed with the XMVB code ${ }^{19,20}$ that includes the covalent and ionic structures with the $6-311++\mathrm{G}^{* *}$ basis set, being thus equivalent to the CAS calculation in the Figure, provides weights equal to $0.88,0.15,-0.03$ for the covalent and two ionic structures, respectively. This has been used as a demonstration of the highly covalent 


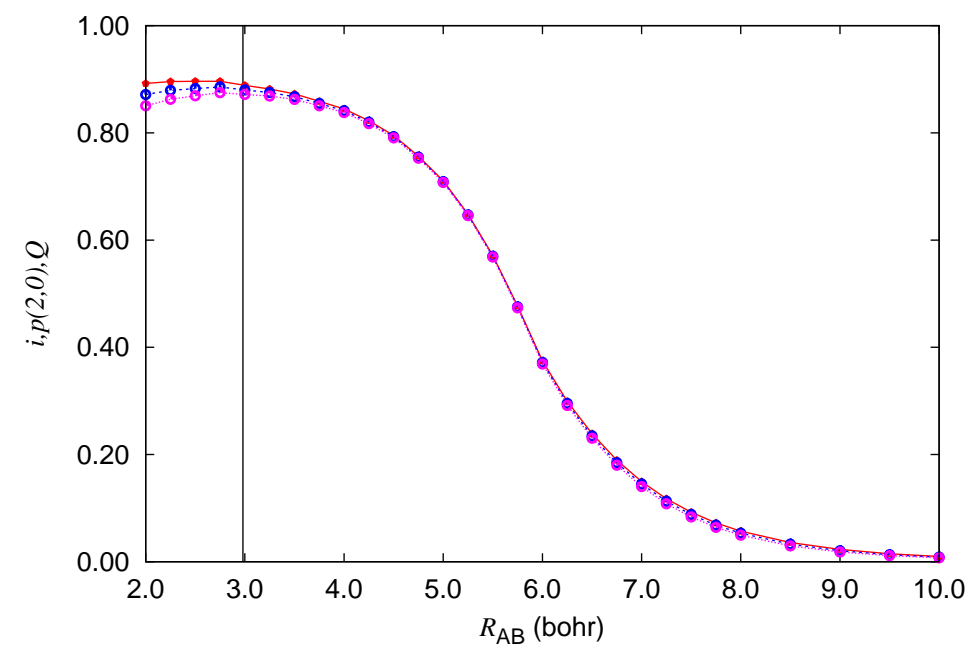

Figure 3: Ionic index $i$ for $\mathrm{LiH}$ (red curve) computed at the CAS[2,2]/6-311++G** level. the $p(2,0)$ component (left is the $\mathrm{H}$ basin) in blue plus the net charge in $\mathrm{Li}$ (in a.u., magenta) are also shown. The equilibrium distance is marked by a vertical line.

nature of the system. In the light of our results, this striking difference is now easy to rationalize. Given the very diffuse character of the $2 s$ orbital of $\mathrm{Li}$, in the covalent VB structure this function invades a large part of the $\mathrm{H}$ atom. Once again, the covalent structure hides a considerable amount of ionic components. In fact, the overlap between the final VB $1 s_{\mathrm{H}}$ and $2 s_{\mathrm{Li}}$ orbitals is -0.69 . In other words, the VB ionic character in $\mathrm{LiH}$ depends on the chosen neutral reference (with largely overlapping $\mathrm{Li}$ and $\mathrm{H}$ atoms), and would be completely different if ionic references were selected. Orbital invariant descriptors lack this problem, and reveal the nature of the discrepancies between $\mathrm{MO}$ and VB interpretations.

Summarizing, we have shown how to read several Valence Bond concepts, like the ionic/covalent character or the covalent-ionic resonance energy from real space chemical bonding descriptors. Electron number distribution functions provide a direct link between VB structures and real space one. Through the relation between EDFs and delocalization indices in simple cases, a straightforward to obtain ionic index has been defined that keeps the physical content of the VB ionic weights. We have also stressed that in real space there is no covalency without fluctuation of the atomic electron populations. In this sense, all covalency emanates from covalent-ionic resonance. This is not so in non-orthogonal VB conventional wisdom because standard covalent structures do 
hide considerable amounts of ionic fluctuation (delocalization). We have shown how this affects VB interpretations in the $\mathrm{H}_{2}$ and $\mathrm{LiH}$ molecules. The large ionic content of the covalent structure in the latter explains why from a VB point of view $\mathrm{LiH}$ is generally considered a covalent molecule. We expect that these insights may help fill the gap between MO, real space, and VB interpretations in chemistry.

We thank the Spanish MINECO, grant CTQ2015-65790-P, the FICyT, grant GRUPIN14-049, and the European Union FEDER for funding.

\section{References}

(1) Gimarc, B. M. Molecular structure and bonding. The qualitative molecular orbital approach; Academic Press: New York, 1979.

(2) Cooper, D. L. Valence Bond Theory; Theoretical and Computational Chemistry; Elsevier Science, 2002.

(3) Gallup, G. A. Valence Bond Methods; Cambridge: New York, 2003.

(4) Shaik, S.; Hiberty, P. A chemist's guide to valence bond theory; John Wiley \& Sons. Hoboken, N.J., 2008.

(5) Shaik, S.; Danovich, D.; Wu, W.; Hiberty, P. C. Nat. Chem. 2009, 1, 443-449.

(6) Bader, R. F. W. Atoms in Molecules; Oxford University Press: Oxford, 1990.

(7) Blanco, M. A.; Martín Pendás, A.; Francisco, E. J. Chem. Theory Comput. 2005, 1, 1096.

(8) Francisco, E.; Martín Pendás, A.; Blanco, M. A. J. Chem. Theory Comput. 2006, 2, 90.

(9) Francisco, E.; Martín Pendás, A.; Blanco, M. A. J. Chem. Phys. 2007, 126, 094102.

(10) Francisco, E.; Martín Pendás, A.; Blanco, M. A. Comp. Phys. Commun. 2008, 178, 621.

(11) Martín Pendás, A.; Francisco, E.; Blanco, M. A. J. Chem. Phys. 2007, 127, 144103. 
(12) Matta, C. F., Boyd,, Eds. The Quantum Theory of Atoms in Molecules: From Solid State to DNA and Drug Design; Wiley-VC, 2007.

(13) Zhang, L.; Ying, F.; Wu, W.; Hiberty, P. C.; Shaik, S. Chem. Eur. J. 2009, 15, 2979.

(14) Wiberg, K. B. Tetrahedron 1968, 24, 1083.

(15) Martín Pendás, A.; Francisco, E.; Blanco, M. A. Phys. Chem. Chem. Phys. 2007, 9, 1087.

(16) Mayer, I. Bond Orders and Energy Components: Extracting Chemical Information from Molecular Wave Functions; CRC Press: Boca Raton, FL, 2016.

(17) García-Revilla, M.; Francisco, E.; Popelier, P. L. A.; Martín Pendás, A. ChemPhysChem 2013, $14,1211$.

(18) Martín Pendás, A.; Francisco, E.; Blanco, M. A. Faraday Discuss. 2007, 135, 423.

(19) Song, L.; Chen, Z.; Song, J.; Chen, .; Su, P.; Mo, Y.; Zhang, Q.; Wu, W.

(20) Song, L.; Mo, Y.; Zhang, Q.; Wu, W. J. Comput. Chem. 2005, 64, 514. 